\title{
Accuracy Evaluation and Sensitivity Analysis of Estimating 3-D Road Centerline Length Using LIDAR and NED
}

This paper proposes an approach to model transportation linear objects in a 3-D space and estimate their 3-D lengths using planimetric road centerline data and elevation data (LIDAR and NED), and quantify the accuracy.

\begin{abstract}
Highway networks are represented by a rich set of linear spatial objects (road segments). Having accurate length information of these linear spatial objects are critical to numerous transportation applications. This paper presents a geographic information system (GIS)-based approach that overlays planimetric road centerline and elevation data to model road centerlines in a 3-D space and calculate their lengths. Elevation data sources included light detection and ranging (LIDAR) and the national elevation dataset (NED). Considering the characteristics of the varying formats of elevation data and highway geometry, two different approaches were proposed to introduce the third dimension (elevation) into the 3-D road model. The accuracies of $\mathrm{d} 3-\mathrm{D}$ distances were evaluated by comparing them to distance measurement instrument (DMI) measured distances. The sensitivity of the length accuracies was analyzed to evaluate the effects from the use of elevation datasets with varying vertical accuracies. The relationship between several geometric properties and the accuracy of the d 3-D distances was also examined. We found that the proposed 3-D approach provides an efficient way to estimate 3-D distances for road centerlines, and by using LIDAR point data, the resulting accuracy of the distances can be improved by over $28 \%$ compared to the use of NED. We also found that certain road geometric properties have direct relationships with the accuracy of the road distances.
\end{abstract}

Keywords: LIDAR, NED, 3-D road modeling, accuracy 


\section{INTRODUCTION}

Highway networks are represented by a rich set of linear spatial objects (road segments). These geo-spatial transportation segments are connected to form topological networks that provide the basis for location referencing systems to locate features like bridges, signs, pavement conditions, and traffic accidents, and can be used to more accurately measure over-the-road travel distances between geographic locations (FGDC 1994). Furthermore, these topological networks can be used to find shortest paths, to determine efficient routes, and to estimate traffic volumes when combined with the variety of network analysis tools.

Transportation data is usually referenced to road networks by using a one-dimensional (1-D) linear referencing model (Quiroga 1999). With this model, objects along a network are located using a set of known points on the network and distances and directions from the known points to the objects along the linear objects. All linear referencing methods are based on this concept (Baker and Blessing 1994), which makes distance critical in transportation spatial databases.

Examining various existing transportation spatial databases reveals that there is no correct and consistent distance information (Rasdorf et al. 2003). The integration of linear referencing data with different linear referencing methods and the integration of linear referencing data with other datasets (global positioning system (GPS) data, for example) reveal that errors vary widely across databases. There is a need to obtain accurate and consistent distance information, and to use this information to update existing transportation spatial databases. This is not a trivial task taking into consideration the number of roads and the mileages of these roads in the United States (Rasdorf et al. 2004).

A number of techniques are available to obtain distances along linear objects. Distance can be extracted from construction and design drawings. This approach suffers from the problems of currency and completeness. A distance measurement instrument (DMI) and an inertial navigation system (INS) might be used to measure the distance directly along lines. The DMI itself is an inexpensive technology consisting of a mechanical device attached to one or more of the wheels of a van and connected to an in-vehicle recorder (Karimi, Hummer, and Khattak 2000). However, a DMI requires frequent calibrations to minimize errors that accumulate as the result of travel. An INS uses accelerometers and gyroscopes to provide pitch, roll, heading information to derive a relative geo-reference for a point. An INS has high accuracy over small distances and is usually more accurate than GPS (depending on types of GPS) but much more costly (Karimi, Hummer, and Khattak 2000). Ground surveying is another technology that can be used to obtain distances along linear objects with a high accuracy when working with straight lines only. GPS is a very promising technique for measuring linear objects. However, the most notable difficulties in using this technology stem from signal blockage in certain areas caused by thick tree canopies or bridges, or multipath problems caused by signal deflection by high-rise buildings.

In addition to the difficulties and limitations mentioned above, all these approaches suffer from a common disadvantage: they are very time-consuming to use to obtain distance information for a large number of roads.

On the other hand, the application of the 3-D spatial modeling approach proposed herein for determining distances provides an efficient way to obtain distance information without field efforts (Quiroga 1999, Rasdorf et al. 2003, Rasdorf et al. 2004). Such an approach could be used to represent road centerlines in a more accurate way, to obtain road centerline distances, and to correct and update distance information in existing transportation spatial databases. The concern is accuracy. Before this 3-D technique can be successfully applied in modeling linear objects in a three-dimensional space and determining their lengths, its accuracy in overlaying planimetric road centerline data with different elevation datasets must be evaluated and quantified. 
This paper presents the results of a research study that focused on estimating 3-D road centerline lengths using LIDAR and NED data and evaluating their accuracy by comparing them to reference data - DMI data in a case study. Four elevation datasets were used: a LIDAR point cloud, a LIDAR 20 foot digital elevation model (DEM), a LIDAR 50 foot DEM, and an NED dataset. The sensitivity of the accuracy to the use of these different elevation datasets with varying vertical accuracies was also analyzed. In addition, the effects on the accuracy from geometric properties of road centerlines were evaluated and reported herein.

\section{METHODOLOGY}

This section briefly describes the 3-D point models used in this research. Based on the chosen model, 3-D distance determination for lines is proposed. In addition, model construction when working with elevation datasets in different formats is discussed.

\section{3-D Point Model}

The key of 3-D spatial modeling is the treatment of the third dimension - elevation in a similar way as the other two planimetric dimensions (Li 1994). From the 2-D aspect, a line object is constructed by connecting two or more vertices. The line segment connecting two neighboring vertices could be a straight line, a circular line, or a polyline depending on the actual characteristics of the line object being constructed and the relative relationship among vertices. Together, the point locations and the connecting line segments compose the geometry of the line under construction.

A natural extension to this principle is to represent a 3-D linear object by connecting two or more 3-D vertices as illustrated in Figure 1. 3-D vertices are points with $\mathrm{X} / \mathrm{Y} / \mathrm{Z}$-coordinates specified in a 3-D space. The line segment connecting two neighboring 3-D vertices could be a straight line, a circular line, or a polyline.

In Figure 1, line segments connecting these vertices can be described via mathematical functions in the format of $\mathrm{Z}=\mathrm{f}(\mathrm{X}, \mathrm{Y})$ and $\mathrm{Y}=\mathrm{f}(\mathrm{X})$, where $\mathrm{X}, \mathrm{Y}$, and $\mathrm{Z}$ are the three dimensions in 3-D GISs and the combination of the two functions together specifies a 3-D line, not a 3-D surface in a three-dimensional space.

In a 2-D GIS, the location of a point is specified in the context of a coordinate system. In Linear Referencing Systems (LRSs), the location of a point along a road segment is specified by a distance along the segment referring to the start point. In other words, only one measure (the distance) is needed to locate a point on a road segment, leading to a one-dimensional model of three-dimensional linear objects.

Taking a similar approach, a 3-D vertex of a road centerline can be located in a threedimensional space via the distance measure (planimetric distance) and the elevation. This leads to a variation of the point model to represent road segments in a 3-D space as illustrated in Figure 2.

With the variant point model proposed above, the lines connecting neighboring 3-D vertices could be described by a function in the format of $Z=f(D)$, where $Z$ is elevation and $D$ is 2-D distance along the line referring to the start point. This variant can be simplified by assuming that all line segments connecting neighboring vertices are straight lines. No mathematical function is necessary to describe the connecting line segments, even though it can be easily derived. This variation requires sufficient number of vertices in order to achieve a high accuracy (Cai 2003). Generally, more 3-D points indicate a closer approximation to the original linear object. 


\section{3-D Distance Determination}

The variation point model described in the previous section may not be desirable in visualization, but it is very convenient for specific 3-D analyses such as distance determination. The 3-D distance determination method in this research was based on this simplified variant, which specifies that a 3-D line consists of 3-D vertices. In addition to its $\mathrm{X} / \mathrm{Y} / \mathrm{Z}$-coordinates, each 3-D vertex has an associated distance that is the planimetric distance along the line from the start point of the line to this vertex. The distance of the straight line segment connecting two neighboring points was calculated as the square root of the sum of the square of the 2-D distance between these two vertices and the square of the elevation difference of these two vertices. The 2-D distance used in this calculation is the difference of the planimetric distances associated with these two vertices. Adding distances of all straight line segments connecting neighboring vertices of a 3-D line leads to the 3-D distance of this 3-D line.

Compared to calculating the planimetric distance between two neighboring vertices using their X/Y-coordinates directly, using the difference in the planimetric distances along the line from two neighboring vertices to the start point as the planimetric distance between two neighboring vertices is more accurate. This is because it takes into consideration the planimetric curvature of the linear objects and is more accurate in 3-D distance determination.

\section{Model Construction}

The critical part in constructing the proposed 3-D point model for lines and carrying out 3-D distance determination is the introduction of the third dimension, or the elevation. More specifically, the concern herein is how to obtain 3-D vertices along road centerlines. Two different approaches were developed in this research to work with elevation datasets in different formats: a snapping approach for working with LIDAR point clouds and a uniform interval and bilinear interpolation approach for working with digital elevation models (DEMs).

\section{Buffering and Snapping Approach}

The buffering and snapping approach for working with point elevation data was developed based on road geometry and point density (Cai and Rasdorf 2008). Point elevation datasets such as LIDAR point clouds have relatively high density. It indicates that lots of points would be on the road pavement surface, even though not many points would lie exactly on the road centerline. Roads are man-made objects with relatively flat surface (small cross-sectional slopes) and consequently, these points on the pavement surface have their elevations very close to the elevations of nearby points along road centerlines and could be used to obtain elevations for 3-D points along road centerlines.

For a given road centerline, a buffer with a size of a half of the road surface width or smaller can be applied to identify elevation points that are on the road surface. Each identified elevation point can then be snapped to the nearest point on the road centerline. These two steps lead to a series of 3-D points along the road centerline.

\section{Uniform Interval and Bilinear Interpolation Approach}

The uniform interval and bilinear interpolation approach used in this research study was designed for obtaining 3-D vertices along lines when working with DEMs (Rasdorf et al. 2004). The uniform interval part identifies the planimetric positions of 3-D vertices on the line while the bilinear interpolation approach introduces elevations to these vertices based on their planimetric positions and the DEMs.

The uniform interval to be used was determined based on the resolution of the DEM data being used. As a general rule, the optimum interval should be the same as the resolution which is 
often represented by one dimension of the cell (for example, 30meters) of the DEM in order to achieve a reasonable accuracy and efficiency.

\section{CASE STUDY}

A transportation case study was designed to implement the developed 3-D approach to estimate 3-D distances for road centerlines, using elevation datasets in different formats with varying vertical accuracies, more specifically, LIDAR point cloud, LIDAR DEMs, and NED. The accuracy of the estimated 3-D lengths was evaluated by comparing them to DMI data. The sensitivity of the accuracy of distance prediction to varying vertical accuracies of elevation datasets was evaluated. In addition, the effects on the accuracy from road centerline geometric properties calculated from the resulting 3-D vertices were assessed.

\section{Study Scope}

The scope of this case study was determined based on the availability of LIDAR data in North Carolina at the time of conducting this research, while considering sample size and variety. The scope of this case study included portions of Interstate highways in 9 counties (Durham, Granville, Halifax, Johnston, Nash, Orange, Vance, Wake, and Wilson), and US and NC routes in Johnston County.

\section{Data Sources}

This case study involved three major datasets: planimetric road centerline data, elevation data for 3-D distance determination (point cloud and DEM), and DMI data for accuracy evaluation.

The planimetric road centerline data were provided by the GIS unit of North Carolina Department of Transportation (NCDOT). It was digitized from 1993 grayscale digital orthophoto quarter-quadrangles (DOQQs) and 1998 color-infrared (CIR) DOQQs with a ground resolution of 1 meter, which were georeferenced to State plane coordinates, using a horizontal datum of North American Datum of 1983 (NAD83).

The elevation data in this case study included LIDAR data and NED. LIDAR data is further categorized into LIDAR point clouds, LIDAR DEMs with a 20-ft resolution, and LIDAR DEMs with a 50-ft resolution. They were obtained from the North Carolina Floodplain Mapping Program (www.ncfloodmaps.com). The obtained LIDAR data is bare earth data free from vegetation, buildings, and other manmade structures, in the format of ASCII files (NCCTS 2003). The horizontal datum is NAD83 and the projection is Lambert Conformal Conic projection. The coordinate system is State Plane (North Carolina). The vertical datum is North American Vertical Datum of 1988 (NAVD88). North Carolina specified a root mean square of error (RMSE) of $20 \mathrm{~cm}$ for coastal counties and a RMSE of $25 \mathrm{~cm}$ for inland counties (NCCTSMP 2001).

The NED is produced by U.S. Geological Survey (USGS) by merging the highest-resolution, best-quality elevation data available across the United States into a seamless raster format (USGS 2003a). It is the result of the USGS effort to provide 1:24,000-scale Digital Elevation Model (DEM) data for the conterminous US and 1:63,360-scale DEM data for Alaska (USGS 2003a, USGS 2003b). In this case study, NED data was obtained from North Carolina State University Spatial Information Lab (http://www.precisionag.ncsu.edu), in the format of interchange files (.e00 files). These interchange files were converted into raster grid files. The horizontal datum is NAD83. The vertical datum is NAVD88. The projection is Lambert Conformal Conic projection. The coordinate system is State plane (North Carolina). The grid spacing of the converted raster data is 1 -arc-second (approximately 30 meters or 98 feet). 
In this case study, the reference data, or DMI data, was collected by researchers for all road segments in the study scope via field work. The DMI used to collect measurements is NITESTAR ${ }^{\circledR}$ Model NS-60 from Nu-Metrics, Inc. This model has a precision of 1 foot/mile (repeatability) in measuring distances (Nu-Metrics 1998). The calibration was done by NCDOT personnel working in the Road Inventory Section of the GIS Unit of NCDOT.

\section{Data Processing}

Based on the previous discussion, 3-D distances were determined for each road segment. When working with LIDAR points, the buffering and snapping approach was used with a buffer at the size of the typical lane width of a particular road segment to ensure that only LIDAR points on road surface were included. When working with a particular DEM dataset, two intervals were taken, one at the full cell size of the DEM and the other at the half cell size of the DEM. Figure 3 illustrates a constructed 3-D road centerline model using LIDAR point data, in which, the vertical scale was exaggerated to show elevation changes. In addition, the DMI distance for each road segment was obtained.

As a result, each road segment is associated with 8 distances, one from using LIDAR point data, one from using LIDAR 20-ft DEMs with a 10-ft interval, one from using LIDAR 20 -ft DEMs with a 20-ft interval, one from using LIDAR 50-ft DEMs with a 25 -ft interval, one from using LIDAR 50-ft DEMs with a 50-ft interval, one from using NED with a 15-meter interval, one from using NED with a 30-meter interval, and the reference distance (DMI measured distance). All combinations of road classes and road centerline distances from different sources were investigated in this study.

\section{Accuracy Evaluation and Sensitivity Analysis}

This section presents the methods used to evaluate the accuracy of the estimated 3-D distance and the sensitivity of the accuracy to elevation datasets with varying format, resolution, and accuracy. Evaluation results are also presented and discussed herein.

\section{General Information}

As stated earlier, each road segment in this case study was associated with $7 \mathrm{~d} 3$-D distances and 1 reference distance (DMI distance). For a particular road segment, error was defined as the difference between an estimated 3-D distance and the reference distance. Proportional error was defined as the error divided by the reference distance. Road segments were categorized based on road types, resulting into four groups: All Road, Interstate, US routes, and NC routes road segments. The group of All Road included Interstate, US routes, and NC routes road segments. There were a total of 28 samples of errors (differences) and a total of 28 samples of proportional errors (proportional differences).

To deal with the problem of misalignment between field county boundary signs and the digital county lines, neighboring road segments touching the same county boundary were combined into one road segment and were counted once in accuracy evaluation.

\section{Accuracy Evaluation and Sensitivity Analysis Results}

RMSE is a commonly used method for spatial accuracy assessment (Daniel and Tennant 2001). The most commonly used RMSEs are 100\% RMSEs calculated from all checkpoints and 95\% RMSEs calculated from the "best" $95 \%$ of the checkpoints that have errors smaller than the errors of the remaining 5\%. Equation (1) below illustrates how RMSE can be calculated.

$$
\operatorname{RMSE}=\left[\left(\mathrm{e}_{1}^{2}+\mathrm{e}_{2}^{2}+\mathrm{e}_{3}^{2}+\ldots+\mathrm{e}_{\mathrm{n}}^{2}\right) / \mathrm{n}\right]^{1 / 2} ; \text { where } \mathrm{e}_{1}, \mathrm{e}_{2}, \mathrm{e}_{3}, \ldots, \mathrm{e}_{\mathrm{n}} \text { are errors. }
$$


Table 1 summarizes $100 \%$ and $95 \%$ RMSEs in this case study. The difference has a unit of foot while the proportional difference has a unit of foot/1,000 feet. A careful review of the discarded 5\% "worst" road segments revealed that the majority were road segments that had either underpass or overpass point(s) as the end(s). This indicates that when adopting the proposed 3-D approach to estimate 3-D road centerline distances, the practitioners must pay extra attention to road segments falling into the scenario of having underpasses or overpasses point(s) as end(s). The sensitivity of the accuracy to elevation datasets with varying formats, resolutions, and vertical accuracies was evaluated by comparing these RMSEs. The trend patterns observed from 95\% RMSEs and 100\% RMSEs were similar and consequently, only graphical comparisons of 95\% RMSEs from the aspects of the difference and proportional difference are presented in Figure 4 and Figure 5, respectively. The shaded area of Table 1 comprises the graphs of Figure 4. The latitudinal axis of Figure 4 lists the 7 data sets and intervals under consideration. In both Figures 4 and 5, straight lines are used to link discrete data points to illustrate trends, if they exist. Since the horizontal axis represents the use of different elevation data sets, it is meaningless to try to interpolate between these discrete data points.

Based on Figure 4, it is observed that for all road segments (diamond), the sample using the LIDAR point data (LP) has the lowest RMSE (the highest accuracy). The RMSEs of samples using LIDAR DEMs are higher than the RMSE of the sample using LIDAR point data, but lower than the RMSEs of the samples using NED. Thus, it would simply appear that LIDAR point cloud data inputs greater accuracy than LIDAR DEMs and they in turn input greater accuracy than NED data sets.

For Interstate road segments and US road segments, the trend lines are close to the trend line of all road segments. The exception is the trend line for NC road segments. The RMSE of the sample using LIDAR point data is higher than the others. However, these RMSEs are too close to each other to reach a meaningful observation of the trend.

Based on Figure 5, it is observed that the trend lines for All Road segments, Interstate Road segments, US Road segments, and NC Road segments are almost parallel to each other. The RMSEs of the samples of a particular road type (but using different elevation datasets and intervals) are close to each other. When all roads are considered, the sample using LIDAR point data has the lowest RMSE. The lowest RMSEs correspond to the samples using LDIAR point data for Interstate Road segments and US Road segments and the samples using LIDAR 20-ft DEMs for NC Road segments.

In all cases, the RMSEs of the samples using NED are imperceptibly higher than the corresponding RMSEs of the samples using LIDAR point data. It is worth pointing out herein that by removing the "worst" 5\% of the data, the improvements on the RMSEs for proportional differences are significant and more significant than those for differences.

Overall, it is observed that when using the same DEM with different interval sizes, the difference between the RMSE values are very small, indicating that the effect from using different intervals on the accuracy of the estimated 3-D distance could be ignored, given these intervals were no larger than the cell resolution. This observation is confirmed by comparing other statistics including means, medians, standard deviations, absolute means (based on error magnitude) in this case study (not included in this paper) (Cai 2003).

\section{Significance Analysis}

The purpose of significance analysis is to evaluate the effects on the accuracy of the estimated 3-D distance from certain geometric properties such as distance, vertical complexity, etc. This section describes the methods being used, the geometric properties under consideration, and evaluation results with observations. 


\section{Analysis Methods}

Two methods were used to evaluate the effects from certain road centerline geometric properties on the accuracy of the estimated distance. The first method was the calculation of sample correlation coefficient of two variables, one was the geometric property under consideration and the other was the error/proportional error in the estimated 3-D distances.

The value of the sample correlation coefficient ranges from -1 to 1 . It could be understood that the closer to \pm 1 the value of the sample correlation coefficient is, the stronger the linear association is. If the value of the sample correlation coefficient is 1 , variables $\mathrm{X}$ and $\mathrm{Y}$ are said to be perfectly correlated (Rao 1998). The sign of the sample correlation coefficient determines if there is a positive or a negative linear association between two variables.

The second method was the grouping and comparison method. With this method, rather than grouping roads into different road types, roads were grouped based on the value of a geometric property under evaluation. RMSEs for these groups were obtained and compared. Given a particular set of estimated 3-D distances determined by the use of elevation dataset and the model parameter, the variation of the RMSEs among the groups could be evaluated and the effects of the factor under consideration on the accuracy of the estimated 3-D distances could be determined.

\section{Geometric Properties under Evaluation}

Geometric properties under evaluation in this research included the actual distance, the average slope and slope change, and the weighted slope and slope change. The distance was defined as the DMI measured distance. The geometric properties related to slopes and slope changes describe the vertical complexity. In this research, most linear objects are relatively long and therefore, it is unreasonable to simply take the start and end points for slope calculation. Rather, two slopes were obtained for each line: the average slope and the weighted average slope. The weight is 3-D distance.

Figure 6 illustrates the calculations of average and weighted slopes and slope changes with a simplified example. Assuming there is a line that consists of three vertices connected by two straight line segments LN1 and LN2, with slopes S1 and S2, respectively:

(1) The average slope is calculated as $(\mathrm{S} 1+\mathrm{S} 2) / 2$.

(2) The weighted slope is calculated as $(\mathrm{S} 1 * \mathrm{D} 1+\mathrm{S} 2 * \mathrm{D} 2) /(\mathrm{D} 1+\mathrm{D} 2)$, where $\mathrm{D} 1$ is the $3-\mathrm{D}$ distance of LN1 and D2 is the 3-D distance of LN2.

(3) The average slope change is calculated as $((\mathrm{S} 1-0)+(\mathrm{S} 2-\mathrm{S} 1)) / 2$.

(4) The weighted slope change is calculated as $((\mathrm{S} 1-0) * \mathrm{D} 1+(\mathrm{S} 2-\mathrm{S} 1) * \mathrm{D} 2) /(\mathrm{D} 1+\mathrm{D} 2)$.

\section{Significance Analysis Results}

This subsection presents the results from significance analysis by using two methods: the sample correlation coefficient approach and the grouping and comparison approach.

\section{(1) Sample Correlation Coefficients}

Table 2 summarizes the results of the sample correlation coefficients based on the difference, the absolute difference (error magnitude), the proportional difference, and the absolute proportional difference (the magnitude of the proportional error) for the geometric properties under evaluation in this research. In Table 2, road types are not under consideration, i.e. only the results for the all road segments are presented. In the original study, road types were under consideration. The observations about Interstate, US, and NC road segments are similar to those of all road segments, and therefore, are not repeated herein (Cai 2003).

It is observed that from the aspect of the proportional difference, there is no linear association between a geometric property under consideration and the accuracy of the estimated 
3-D distance, as indicated by the close to 0 sample correlation coefficients. However, when the error magnitudes (the absolute difference and the absolute proportional difference) are considered, all five geometric properties under consideration in this research show a more significant linear association with the accuracy of the estimated 3-D distance, as indicated by the relatively larger sample correlation coefficient magnitudes. The correlation coefficient signs indicate a positive linear association between a geometric property under consideration in this research and the error of the estimated 3-D distance but a negative linear association from the aspect of the proportional error.

The exact meaning of the positive or negative linear association is demonstrated with an example: the average slope has a positive linear association with the error but a negative linear association with the proportional error of the estimated 3-D distance. In other words, the steeper the average slope of the road segment is, the larger the error it has and the smaller the proportional error it has in its estimated 3-D distance.

\section{(2) Grouping and Comparison}

Table 3 illustrates the designed groups based on the distance. Group 1 was used to include all very short Road segments. Group 7 was used to include all very long Road segments. The symbol "(" means "greater than". The symbol ")" means "less than". The symbol "]" means "less than or equal to".

Table 4 summarizes the RMSEs based on the difference and the proportional difference of these groups. Figures 7 and 8 graphically illustrate the comparisons of these RMSEs from the aspect of the difference and the proportional difference, respectively.

It is observed from Figure 7 that the general trend of the RMSEs indicates that the longer the distance, the larger the RMSE, when using a particular combination of an elevation dataset and an interval size. The two groups having the shortest distances are the two groups having the smallest RMSEs. The two groups having the longest distances are the two groups having the largest RMSEs. The other three groups having intermediate distances are the groups having intermediate RMSEs.

From Figure 8, it is obvious that the seven trend lines corresponding to the use of seven different combinations of elevation datasets and model parameters are clustered together. The general trend for these seven trend lines indicated that groups having longer distances are associated with smaller RMSEs of the proportional difference while groups having shorter distances are associated with larger RMSEs of the proportional difference.

These observations confirm the linear associations observed based on sample correlation coefficients. The same analysis was conducted for the factors of average slope, weighted average slope, average slope change, and weighted slope change. Similar observations were obtained, which confirmed the linear associations observed from the sample correlation coefficients. In other words, there is a general positive linear association between a factor under consideration and the error and there is a general negative linear association between a factor under consideration and the proportional error. With the aid of these observations, road segments with particular geometric properties could be identified as those that request additional attention when adopting the proposed 3-D approach to estimate 3-D distances for road centerlines.

\section{CONCLUSIONS AND DISCUSSION}

This paper observes that the accuracy of the calculated 3-D distances varies with the use of different elevation datasets. From the aspect of differences (error magnitude) using the 100\% RMSE as the measure of the accuracy, the use of LIDAR point data in this case study improved the accuracy by about $28 \%$ compared to the use of NED data. The use of LIDAR DEMs improved the accuracy by about $6 \%$ compared to the use of NED data. Based upon the $95 \%$ 
RMSE, the use of LIDAR point data in this case study improved the accuracy by about $25 \%$ compared to the use of NED data. The use of LIDAR DEMs improved the accuracy by about $9 \%$ compared to the use of NED data.

From the aspect of proportional differences (proportional error magnitude), the improvements due to the use of higher accurate elevation datasets were not as significant as those shown for differences above. This was the case because $43 \%$ of the road segments in the case study were longer than $10,000 \mathrm{ft}, 53 \%$ were longer than $5,000 \mathrm{ft}$, and about $73 \%$ were longer than $1,000 \mathrm{ft}$ and longer road segments have a higher proportional accuracy. The 3-D distances calculated using LIDAR point data with the snapping approach in this research had the highest accuracy when compared to DMI measured distances.

Observations and discussions provided above provide guidance for practitioners on selecting appropriate elevation data sets to carry out road distance estimating and updating to meet accuracy needs. The RMSE values obtained in this study also identify the accuracy range of estimated road distances, using the proposed 3-D approach with different elevation data sets with varying accuracy and precision. Thus, practitioners can determine whether the proposed approach with the use of readily available NED data meets their application accuracy needs and whether it is desirable or necessary to replace NED data with more expensive LIDAR data.

While the accuracy of the estimated 3-D distances is dependent on the elevation dataset being used, if the same elevation dataset is used, but two different intervals are taken (one equivalent to half of the cell size and the other equivalent to the full cell size of the elevation data), the accuracies of the estimated 3-D distances are almost the same. In other words, the accuracy of the estimated 3-D distances is not dependent on the interval sizes if they are less than or equal to the cell size of the elevation data. The accuracy of the estimated 3-D distances also varies with road types without discernible pattern. This leads to the recommendation that when adopting the proposed 3-D approach to estimate road centerline distance using DEMs, the optimum interval ought to be taken at a value that is equivalent to the DEM cell size (resolution) to achieve the greatest accuracy while minimizing the computing time and resource consumption.

It is also concluded that the geometric properties under consideration in this study (the distance, the average and weighted slope, and the average and weighted slope change) have close relationships to the accuracy of the estimated 3-D distance. The distance factors capture the vertical curvature sinuousness. There is a positive association between a geometric property and the error but a negative association from the aspect of the proportional error. Thus, these geometric properties become "accuracy indicators" of the estimated 3-D road centerline distance. These accuracy-indicating geometric properties provide a quick accuracy estimate of a particular estimated road distance. They also help identifying highly vulnerable road segments (groups of road segments with a great potential for big estimate errors) in adopting the proposed 3-D approach in distance estimate and consequently, appropriate quality control caution can be applied to improve the accuracy in road distance estimating.

Some key findings are emphasized herein.

1) The 3-D modeling approach developed in this research provides an efficient way to estimate 3-D distances for road centerlines. The designed case study quantified the impacts from elevation data with varying accuracy to the accuracy of road distance estimates. The case study also identified a particular group of road segments that occupy certain characteristics of road geometries and require extra caution when adopting the proposed approach to build a more accurate road centerline model.

2) The preference order of the elevation datasets to be used in modeling road centerlines as 3-D lines and estimating their distances is LIDAR point data, LIDAR DEMs, and 
NED (from higher preference to lower preference). LIDAR point data are the most preferred elevation dataset to be used.

3) Using this approach, all distances are calculated by computer. For most practical applications no DMI, ground surveying, or GPS measurement is needed to determine road centerline distance. This indicates significant savings in equipment, time, and personnel. The implications of the work, however, transcend the transportation application investigated herein. In fact, the results apply to any linear entity that can be modeled using GIS, particularly infrastructure applications including water, sewer, other forms of piping, communication and power lines, etc.

\section{ACKNOWLEDGEMENT}

The authors are grateful to the National Science Foundation (NSF) for funding portions of this work through NSF Grant No. 9978592. The authors would also like to acknowledge the many contributions of the NCDOT to this work, particularly the personnel of the Information Technology Branch and the GIS Unit.

\section{REFERENCES}

Baker, W. T. and Blessing, W. E., 1994. NCHRP Synthesis of Highway Practice 21: Highway Location Reference Methods, Transportation Research Board, National Research Council, Washington, D. C., Pages 29.

Cai, H.,2003. Accuracy Evaluation of a 3-D Spatial Modeling Approach to Model Linear Objects and Predict their Lengths, PhD Dissertation, Department of Civil Engineering, North Carolina State University, Raleigh, NC, November.

Cai, H and Rasdorf, W. 2008, "Modeling Road Centerlines and Predicting Lengths in 3-D Using LIDAR Point Cloud and Planimetric Road Centerline Data," Computer-Aided Civil and Infrastructure Engineering, 23, Pages 157-173, April.

Daniel, C. and K. Tennant, 2001. DEM Quality Assessment, Digital Elevation Model Technologies and Applications: the DEM Users Manual, American society for Photogrammetry and Remote Sensing, Bethesda, MA.

Federal Geographic Data Committee (FGDC), 1994. Subcommittee Reports: FGDC Ground Transportation Subcommittee Positions and Recommendations on Linear Referencing Systems, FGDC Ground Transportation Subcommittee.

Federal Geographic Data Committee (FGDC), 1999. National Spatial Data Infrastructure Framework Transportation Identification Standard - Working Draft, FGDC Ground Transportation Subcommittee, May.

Karimi, H. A., Khattak, A. J., and Hummer, J. E., 2000. "Evaluation of Mobile Mapping Systems for Roadway Data Collection," Journal of Computing in Civil Engineering, American Society of Civil Engineers, Volume 14, Number 3, Pages 168-173, July.

Li, R., 1994. "Data Structures and Application Issues in 3-D Geographic Information Systems," Geomatica, Volume 48, Number 3, Pages 209-224.

Maune, D., 2001. Digital Elevation Model Technologies and Applications: The DEM Users Manual, Editor Maune, F. D., American Society for Photogrammetry and Remote Sensing, 5410 Grosvenor Lane, Suite 210, Bethesda, ML 20814.

NCCTS, 2003. "LIDAR and Digital Elevation Data Fact Sheet," North Carolina Cooperating Technical State, http://www.ncfloodmaps.com/pubdocs/lidar_final_jan03.pdf, January 2003, Accessed May 2003.

NCCTSMP, 2001. "Issue 5: Quality Control of LIDAR Elevation Data in North Carolina, http://www.ncgs.state.nc.us/flood/ip05 nc lidar qc.dbf, October 2001, Accessed May 2003.

Nu-Metrics, Inc., 2002. http://www.nu-metrics.com/ pdf/nite_man_2002.pdf, Copyright Nu-Metrics 2002, Accessed March 6.

Nu-Metrics, Inc., 1998. NITESTAR ${ }^{\circledR}$, Accurate Distance Measuring from Any Vehicle, Model No. NS50/NS-60, Nu-Metrics, Inc. Uniontown, PA 15401. 
Quiroga, C. A., 1999. "Accuracy of Linearly Referenced Data by Using Geographic Information Systems," Transportation Research Record 1660, Transportation Research Board, National Research Council, Washington, D. C., Pages 100-107.

Rao, P. V., 1998. Statistical Research Methods in the Life Sciences, Brooks/Cole Publishing Company, 511 Forest Lodge Road, Pacific Grove, CA 93950.

Rasdorf, W., Cai, H., Tilley, C., Brun, S., Karimi, H., and Robson, F., 2003. "Transportation Distance Measurement Data Quality," Journal of Computing in Civil Engineering, American Society of Civil Engineers, Volume 17, Number 2, Pages 75-87, April.

Rasdorf, W., Cai, H., Tilley, C., Brun, S., and Robson, F., 2004. "Accuracy Assessment of Interstate Highway Length Measurement Using DEM," Journal of Surveying Engineering, American Society of Civil Engineers, Volume 130, Number 3, Pages 142-150, August.

USGS, 2003a, "USGS Digital Elevation Data Model," http://edcwww.cr.usgs.gov/glis/hyper/guide/usgs dem, Accessed March, 2004.

USGS, 2003b, "National Elevation Dataset," November 2003, http://gisdata.usgs.net/ned/, Accessed March 26, 2003. 


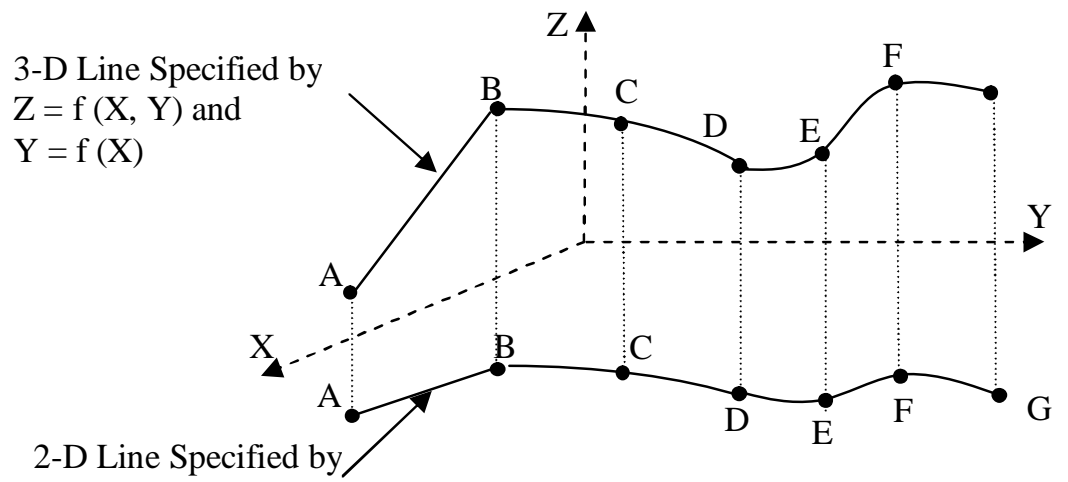
$\mathrm{Y}=\mathrm{f}(\mathrm{X})$

Figure 1 Line Construction in a 3-D GIS

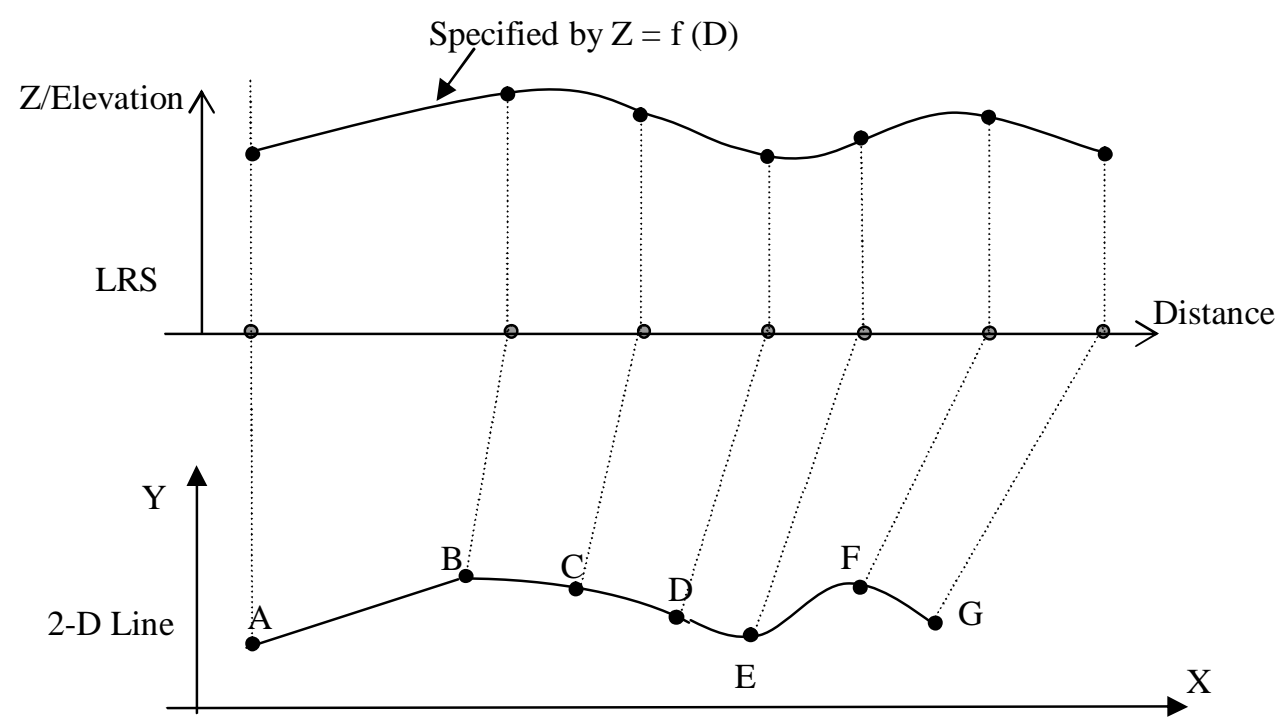

Figure 2 An LRS-Based Variation of the 3-D Point Model (Modified after Cai and Rasdorf 2008) 


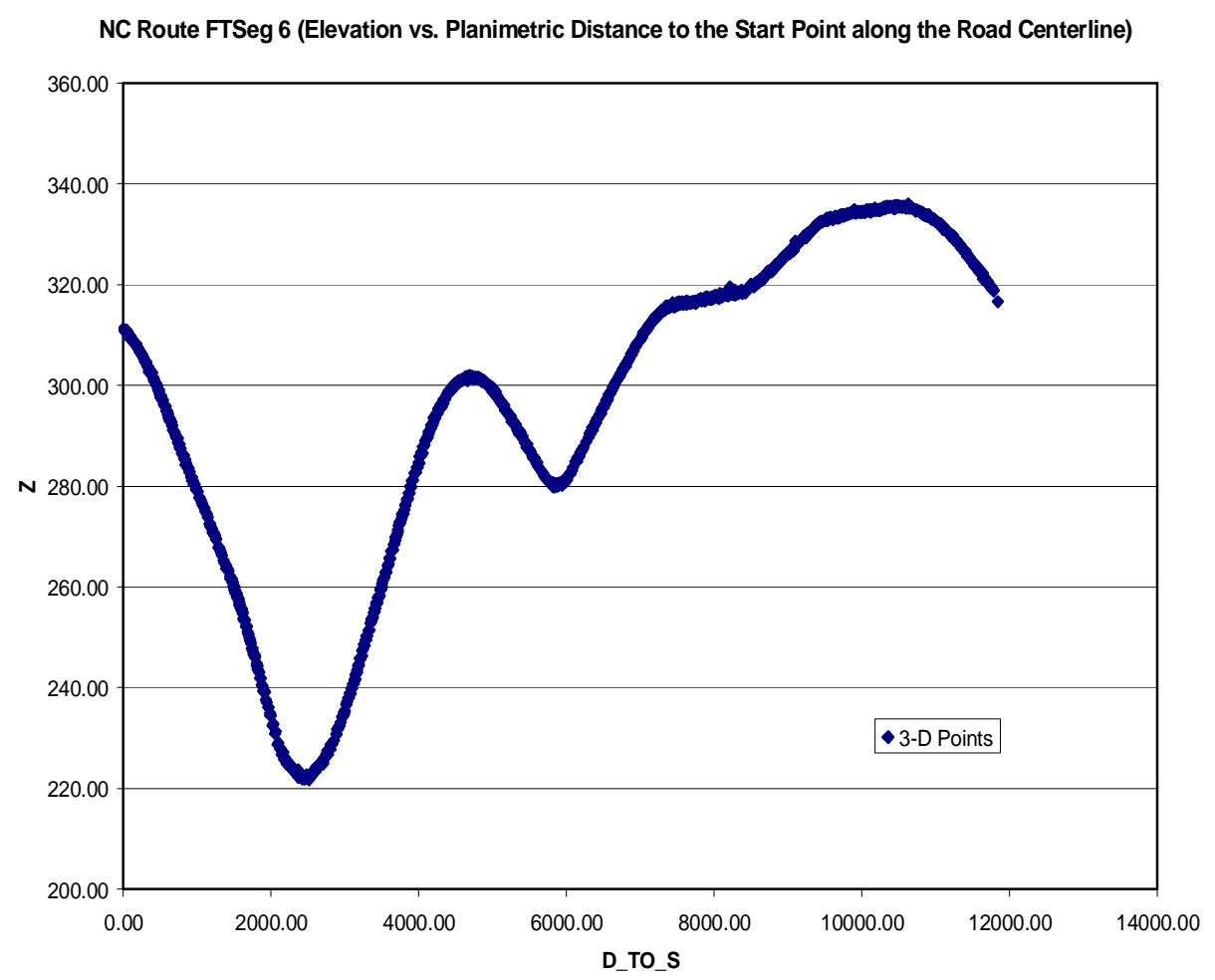

Figure 3 An Example of Constructed 3-D Road Centerline Model

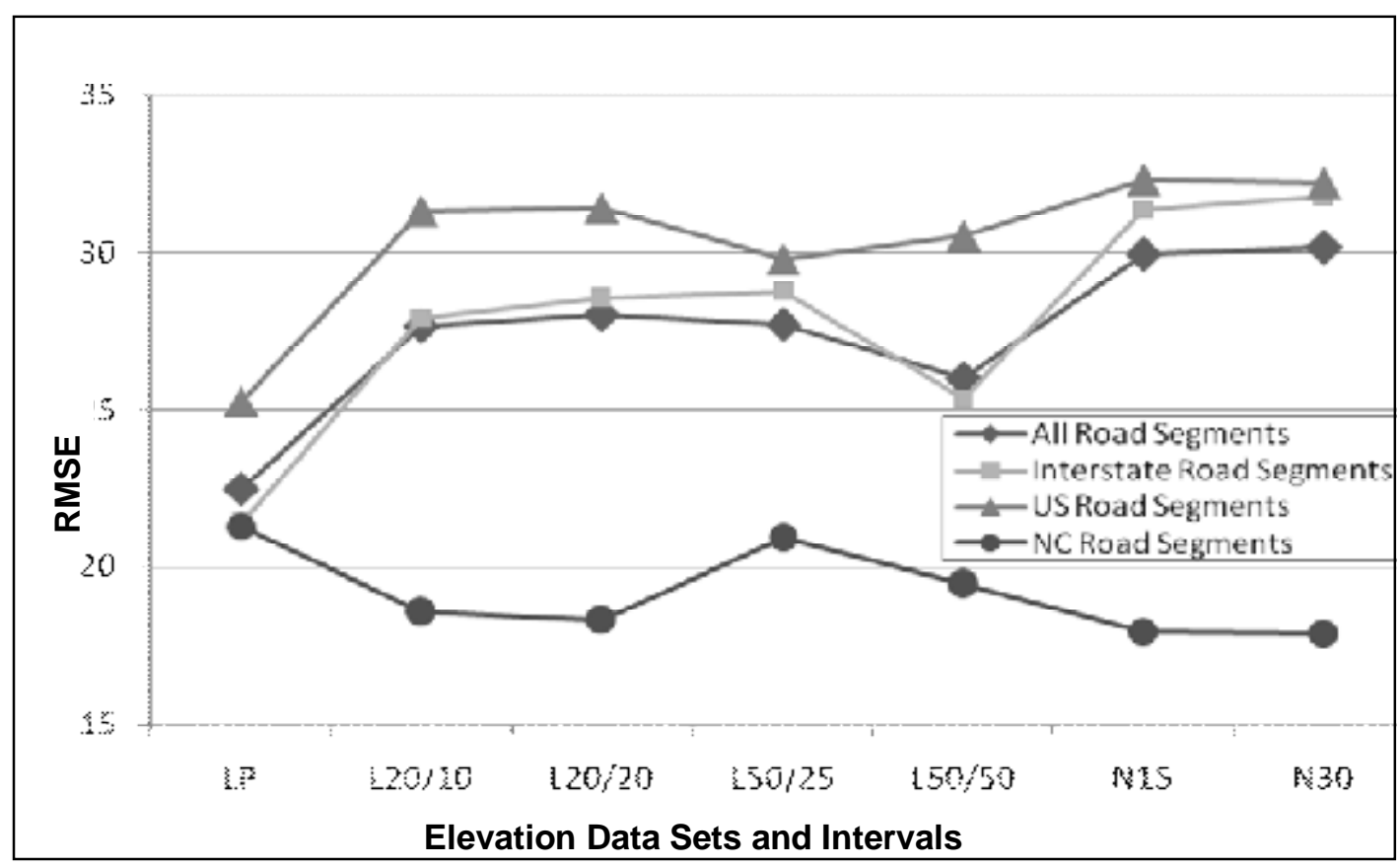

Figure 4 Comparisons of 95\% RMSEs from the Aspect of the Difference 


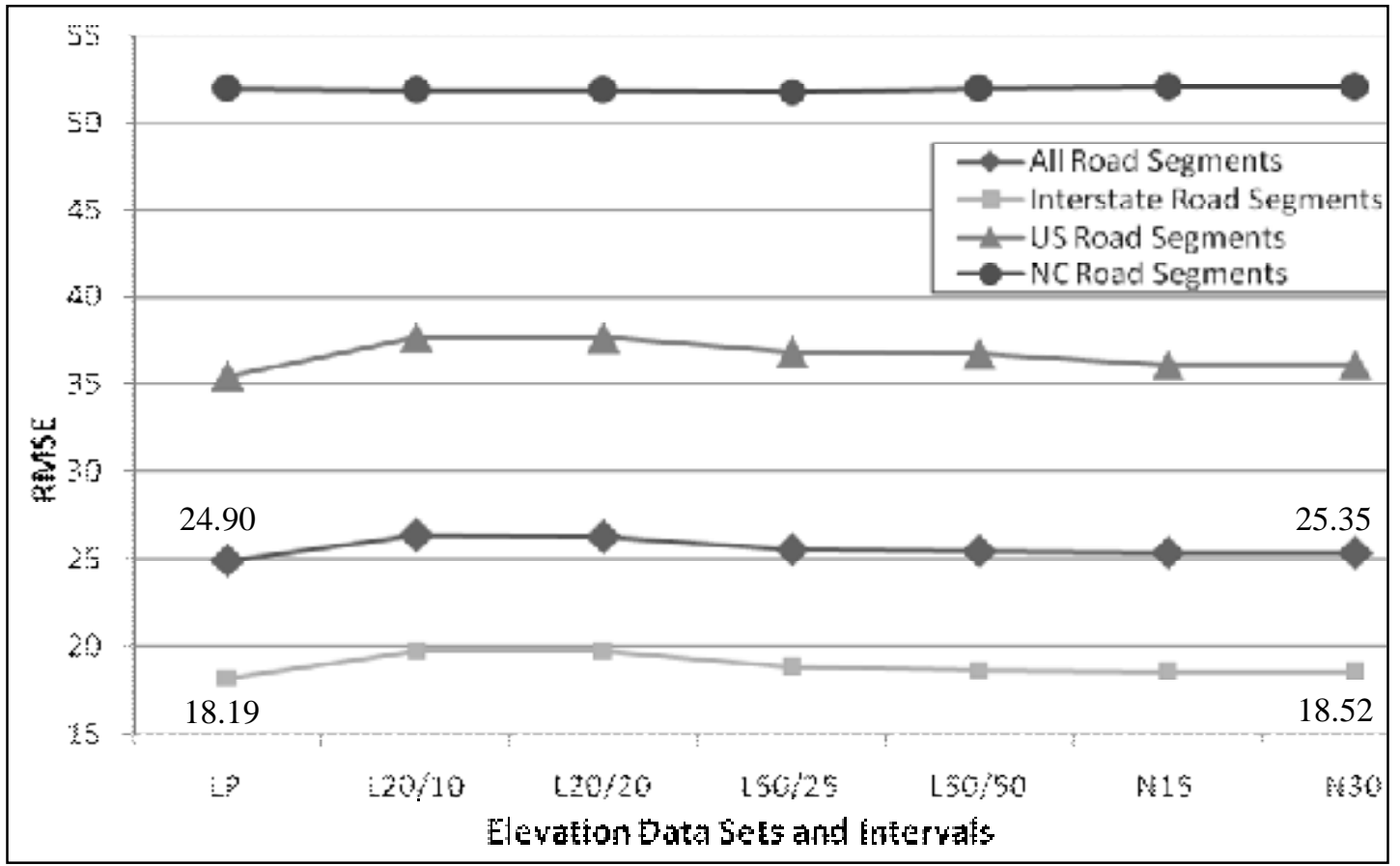

Figure 5 Comparisons of 95\% RMSEs from the Aspect of Proportional Difference

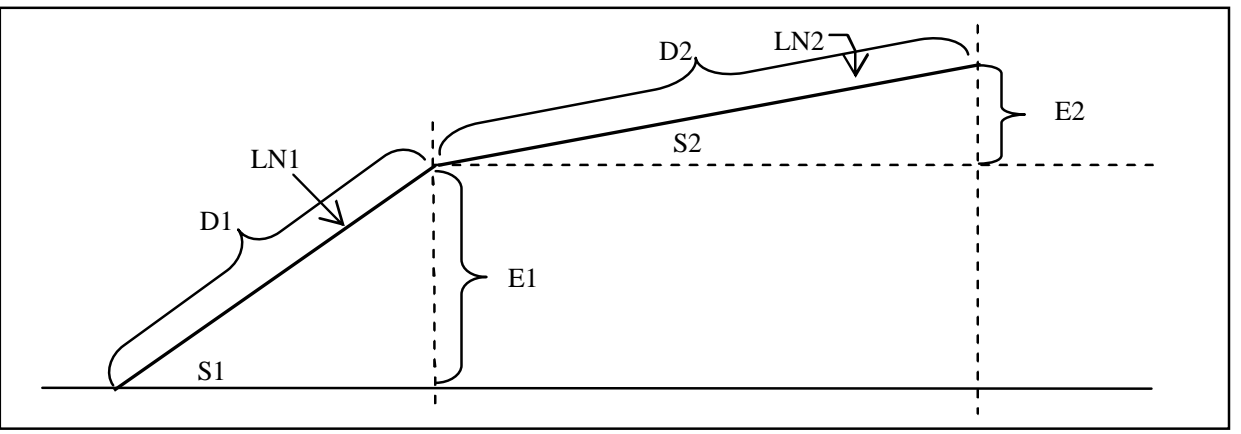

Figure 6 Illustrations of Slope and Slope Change Calculation 


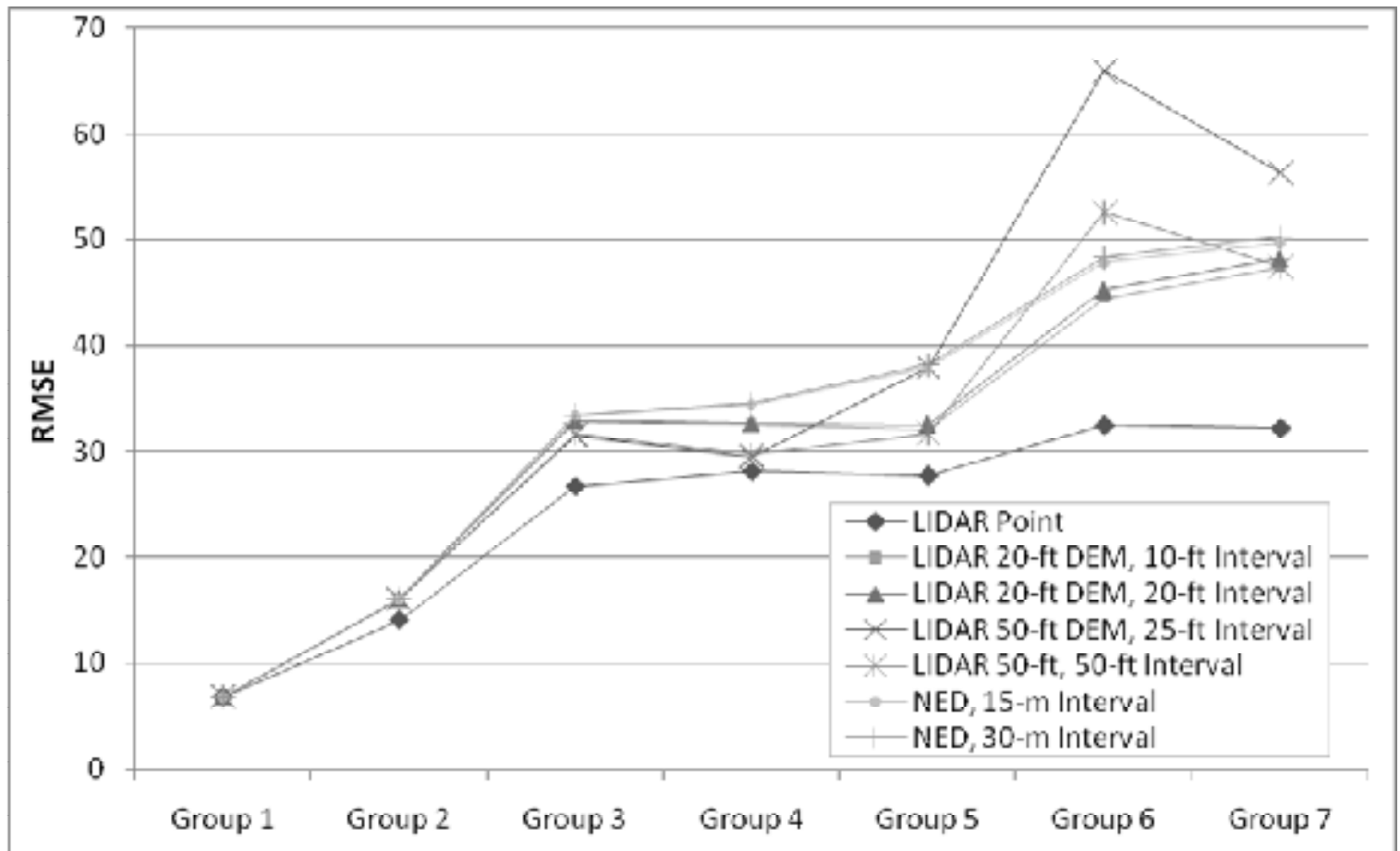

Figure 7 Comparisons of RMSEs of the Difference for Groups Based on Their Length

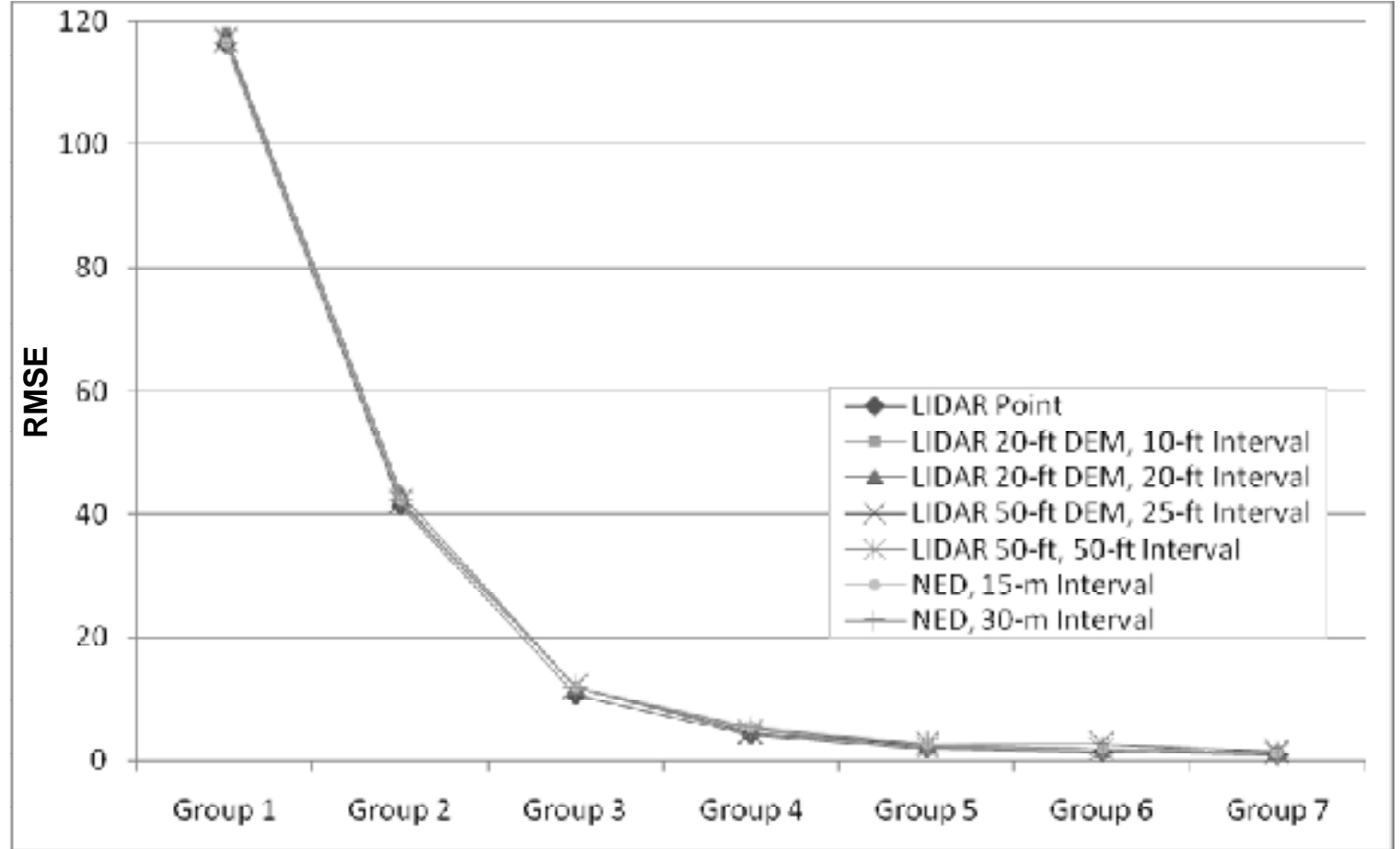

Figure 8 Comparisons of RMSEs of the Proportional Difference for Groups Based on Their

\section{Length}


Table 1 Summary of RMSEs

\begin{tabular}{|c|c|c|c|c|c|c|c|c|c|}
\hline \multirow{2}{*}{ RMSE } & \multirow{2}{*}{$\begin{array}{l}\text { Error } \\
\text { Format }\end{array}$} & \multirow{2}{*}{ Road Type } & \multirow{2}{*}{$\begin{array}{c}\text { LIDAR } \\
\text { Point } \\
\text { Data }\end{array}$} & \multicolumn{2}{|c|}{$\begin{array}{c}\text { LIDAR 20-ft } \\
\text { DEM }\end{array}$} & \multicolumn{2}{|c|}{$\begin{array}{c}\text { LIDAR 50-ft } \\
\text { DEM }\end{array}$} & \multicolumn{2}{|c|}{ NED } \\
\hline & & & & $\begin{array}{c}\text { 10-ft } \\
\text { Interval }\end{array}$ & $\begin{array}{c}20-f t \\
\text { Interval }\end{array}$ & $\begin{array}{c}25-f t \\
\text { Interval }\end{array}$ & $\begin{array}{c}\text { 50-ft } \\
\text { Interval }\end{array}$ & $\begin{array}{c}15-m \\
\text { Interval }\end{array}$ & $\begin{array}{c}\text { 30-m } \\
\text { Interval }\end{array}$ \\
\hline \multirow{8}{*}{$\begin{array}{l}\text { 100\% } \\
\text { RMSE }\end{array}$} & \multirow{4}{*}{ Difference } & "All Road segments & 25.65 & 333.30 & $\begin{array}{l}33.73 \\
\end{array}$ & 33.90 & $\begin{array}{l}34.16 \\
\end{array}$ & 30.53 & 35.74 \\
\hline & & $\begin{array}{c}\text { Interstate Road } \\
\text { segments }\end{array}$ & 24.76 & 33.38 & 33.99 & 35.84 & 35.83 & 36.43 & 36.83 \\
\hline & & $\begin{array}{l}\text { US Road segments } \\
\end{array}$ & 28.26 & 37.39 & 37.62 & 33.83 & 35.04 & 38.69 & 38.53 \\
\hline & & NC Road segments & 24.04 & 22.69 & 22.62 & 23.99 & 23.05 & 22.76 & 22.79 \\
\hline & \multirow{4}{*}{$\begin{array}{l}\text { Proportional } \\
\text { Difference }\end{array}$} & All Road segments & 50.44 & $\begin{array}{l}51.28 \\
\end{array}$ & 51.26 & 50.79 & 50.75 & 50.68 & 50.68 \\
\hline & & $\begin{array}{c}\text { Interstate Road } \\
\text { segments }\end{array}$ & 32.06 & 33.16 & 33.12 & 32.33 & 32.24 & 32.29 & 32.29 \\
\hline & & US Road segments & 63.72 & 64.99 & 64.98 & 64.51 & 64.45 & 64.14 & 64.14 \\
\hline & & NC Road segments & 78.80 & 78.74 & 78.74 & 78.71 & 78.78 & 78.83 & 78.83 \\
\hline \multirow{8}{*}{$\begin{array}{l}95 \% \\
\text { RMSE }\end{array}$} & \multirow{4}{*}{ Difference } & All Road segments & 22.48 & 27.65 & 28.01 & 27.71 & 26.01 & 29.95 & 30.16 \\
\hline & & $\begin{array}{c}\begin{array}{c}\text { Interstate Road } \\
\text { segments }\end{array} \\
\end{array}$ & 21.39 & 27.95 & 28.53 & 28.75 & 25.38 & 31.38 & 31.77 \\
\hline & & US Road segments & 25.28 & 31.31 & 31.42 & 29.83 & 30.51 & 32.29 & 32.21 \\
\hline & & NC Road segments & 21.32 & 18.56 & 18.31 & 20.91 & 19.47 & 17.98 & 17.92 \\
\hline & \multirow{4}{*}{$\begin{array}{l}\text { Proportional } \\
\text { Difference }\end{array}$} & All Road segments & 24.90 & 26.36 & 26.33 & 25.58 & 25.45 & 25.35 & 25.35 \\
\hline & & $\begin{array}{c}\text { Interstate Road } \\
\text { segments }\end{array}$ & 18.19 & 19.76 & 19.75 & 18.80 & 18.64 & 18.52 & 18.52 \\
\hline & & US Road segments & 35.47 & 37.73 & 37.72 & 36.87 & 36.76 & 36.19 & 36.19 \\
\hline & & NC Road segments & 51.93 & 51.82 & 51.82 & 51.78 & 51.89 & 52.02 & 52.02 \\
\hline
\end{tabular}

Table 2 Summary of Sample Correlation Coefficients

\begin{tabular}{|c|c|c|c|c|c|c|c|c|}
\hline \multirow{2}{*}{$\begin{array}{l}\text { Geometric } \\
\text { Property }\end{array}$} & \multirow{2}{*}{ Error Format } & \multirow{2}{*}{$\begin{array}{c}\text { LIDAR } \\
\text { Point } \\
\text { Data }\end{array}$} & \multicolumn{2}{|c|}{$\begin{array}{c}\text { LIDAR 20-ft } \\
\text { DEM }\end{array}$} & \multicolumn{2}{|c|}{$\begin{array}{c}\text { LIDAR 50-ft } \\
\text { DEM }\end{array}$} & \multicolumn{2}{|c|}{ NED } \\
\hline & & & $\begin{array}{c}10-\mathrm{ft} \\
\text { Interval }\end{array}$ & $\begin{array}{c}20-f t \\
\text { Interval }\end{array}$ & $\begin{array}{c}\text { 25-ft } \\
\text { Interval }\end{array}$ & $\begin{array}{c}50-f t \\
\text { Interval }\end{array}$ & $\begin{array}{c}\text { 15-m } \\
\text { Interval }\end{array}$ & $\begin{array}{c}\text { 30-m } \\
\text { Interval }\end{array}$ \\
\hline \multirow{4}{*}{ Distance } & Difference & -0.07 & -0.34 & -0.36 & 0.16 & 0.06 & -0.35 & -0.37 \\
\hline & Absolute Difference & 0.31 & 0.36 & 0.37 & 0.35 & 0.34 & 0.40 & 0.41 \\
\hline & Proportional Difference & 0.10 & 0.09 & 0.09 & 0.09 & 0.09 & 0.10 & 0.10 \\
\hline & Absolute Proportional Difference & -0.38 & -0.39 & -0.38 & -0.38 & -0.38 & -0.38 & -0.38 \\
\hline \multirow{4}{*}{$\begin{array}{l}\text { Average } \\
\text { Slope }\end{array}$} & Difference & -0.22 & -0.33 & -0.33 & -0.15 & -0.20 & -0.34 & -0.34 \\
\hline & Absolute Difference & 0.24 & 0.36 & 0.36 & 0.19 & 0.25 & 0.36 & 0.36 \\
\hline & Proportional Difference & 0.00 & -0.05 & -0.05 & -0.04 & -0.04 & -0.04 & -0.04 \\
\hline & Absolute Proportional Difference & -0.25 & -0.23 & -0.23 & -0.23 & -0.22 & -0.22 & -0.22 \\
\hline \multirow{4}{*}{$\begin{array}{l}\text { Weighted } \\
\text { Slope }\end{array}$} & Difference & -0.19 & -0.31 & -0.31 & -0.14 & -0.19 & -0.32 & -0.32 \\
\hline & Absolute Difference & 0.20 & 0.33 & 0.33 & 0.17 & 0.23 & 0.34 & 0.34 \\
\hline & Proportional Difference & -0.03 & -0.07 & -0.07 & -0.06 & -0.07 & -0.07 & -0.07 \\
\hline & Absolute Proportional Difference & -0.21 & -0.20 & -0.20 & -0.20 & -0.19 & -0.19 & -0.19 \\
\hline \multirow{4}{*}{$\begin{array}{c}\text { Average } \\
\text { Slope Change }\end{array}$} & Difference & -0.24 & -0.36 & -0.36 & -0.15 & -0.21 & -0.37 & -0.37 \\
\hline & Absolute Difference & 0.26 & 0.39 & 0.39 & 0.22 & 0.29 & 0.40 & 0.40 \\
\hline & Proportional Difference & 0.02 & -0.02 & -0.02 & -0.02 & -0.02 & -0.01 & -0.01 \\
\hline & Absolute Proportional Difference & -0.30 & -0.29 & -0.29 & -0.28 & -0.28 & -0.27 & -0.27 \\
\hline \multirow{4}{*}{$\begin{array}{c}\text { Weighted } \\
\text { Slope Change }\end{array}$} & Difference & -0.25 & -0.39 & -0.39 & -0.17 & -0.23 & -0.39 & -0.39 \\
\hline & Absolute Difference & 0.27 & 0.41 & 0.41 & 0.23 & 0.29 & 0.42 & 0.42 \\
\hline & Proportional Difference & 0.00 & -0.05 & -0.05 & -0.04 & -0.04 & -0.04 & -0.04 \\
\hline & Absolute Proportional Difference & -0.30 & -0.28 & -0.28 & -0.28 & -0.27 & -0.27 & -0.27 \\
\hline
\end{tabular}


Table 3 Illustrations of Groups Based on the Distance

\begin{tabular}{|c|c|c|c||}
\hline \hline Group Name & Distance Range (ft) & $\begin{array}{c}\text { Number of Road } \\
\text { segments }\end{array}$ & Percentage \\
\hline \hline Group 1 & $(0,100]$ & 46 & $17.36 \%$ \\
\hline Group 2 & $(100,1,000]$ & 26 & $9.81 \%$ \\
\hline Group 3 & $(1,000,5,000]$ & 52 & $19.62 \%$ \\
\hline Group 4 & $(5,000,10,000]$ & 28 & $10.57 \%$ \\
\hline Group 5 & $(10,000,20,000]$ & 38 & $14.34 \%$ \\
\hline Group 6 & $(20,000,30,000]$ & 32 & $12.08 \%$ \\
\hline Group 7 & $(30,000,+\infty)$ & 43 & $16.23 \%$ \\
\hline Total & -- & 265 & $100 \%$ \\
\hline \hline
\end{tabular}

Table 4 Summary of RMSEs for Groups Based on the Distance

\begin{tabular}{|c|c|c|c|c|c|c|c|c|}
\hline \multirow{2}{*}{$\begin{array}{c}\text { Error } \\
\text { Format }\end{array}$} & \multirow[b]{2}{*}{ Group } & \multirow{2}{*}{$\begin{array}{c}\text { LIDAR } \\
\text { Point } \\
\text { Data } \\
\end{array}$} & \multicolumn{2}{|c|}{ LIDAR 20-ft DEM } & \multicolumn{2}{|c|}{ LIDAR 50-ft DEM } & \multicolumn{2}{|c|}{ NED } \\
\hline & & & $\begin{array}{c}10-\mathrm{ft} \\
\text { Interval }\end{array}$ & $\begin{array}{c}20-f t \\
\text { Interval }\end{array}$ & $\begin{array}{c}25-\mathrm{ft} \\
\text { Interval }\end{array}$ & $\begin{array}{c}50-f t \\
\text { Interval }\end{array}$ & $\begin{array}{c}15-m \\
\text { Interval }\end{array}$ & $\begin{array}{c}\text { 30-m } \\
\text { Interval }\end{array}$ \\
\hline \multirow{7}{*}{ 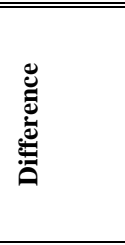 } & Group 1 & 6.75 & 6.84 & 6.84 & 6.78 & 6.78 & 6.77 & 6.77 \\
\hline & Group 2 & 14.10 & 16.11 & 16.12 & 16.06 & 16.07 & 16.03 & 16.04 \\
\hline & Group 3 & 26.73 & 32.80 & 32.93 & 31.56 & 31.63 & 33.39 & 33.42 \\
\hline & Group 4 & 28.15 & 32.56 & 32.71 & 29.45 & 29.70 & 34.48 & 34.53 \\
\hline & Group 5 & 27.76 & 32.19 & 32.62 & 37.92 & 31.69 & 37.95 & 38.10 \\
\hline & Group 6 & 32.52 & 44.51 & 45.30 & 65.97 & 52.60 & 47.93 & 48.29 \\
\hline & Group 7 & 32.23 & 47.40 & 48.24 & 56.33 & 47.48 & 49.72 & 50.20 \\
\hline \multirow{7}{*}{ 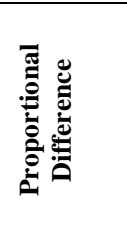 } & Group 1 & 116.40 & 117.94 & 117.90 & 116.96 & 116.92 & 116.61 & 116.61 \\
\hline & Group 2 & 41.30 & 43.25 & 43.19 & 42.00 & 41.83 & 42.27 & 42.27 \\
\hline & Group 3 & 10.54 & 11.83 & 11.84 & 11.87 & 11.86 & 11.95 & 11.96 \\
\hline & Group 4 & 4.23 & 5.06 & 5.09 & 4.46 & 4.61 & 5.40 & 5.41 \\
\hline & Group 5 & 1.95 & 2.27 & 2.29 & 2.60 & 2.22 & 2.62 & 2.63 \\
\hline & Group 6 & 1.32 & 1.76 & 1.79 & 2.51 & 1.98 & 1.92 & 1.93 \\
\hline & Group 7 & 0.80 & 1.22 & 1.24 & 1.35 & 1.17 & 1.23 & 1.24 \\
\hline
\end{tabular}

